\title{
Helicobacter pylori infection transmitted sexually via oral-genital contact: a hypothetical model
} Hypothesis

\author{
Guy D Eslick
}

Objectives: To postulate that Helicobacter pylori infection may be transmitted sexually, using the human female vagina as a reservoir for transmission.

Methods: A literature search was performed using Medline, Embase, Biological Abstracts, and Currents Contents (January 1983 to August 2000). Relevant keywords were used and additional manual searches were made using the reference lists from the selected articles to retrieve other papers relevant to the topic.

Conclusions: Helicobacter pylori infection could be transmitted sexually with the vagina acting as a potential temporary/permanent reservoir given the right environmental conditions. (Sex Transm Inf 2000;76:489-492)

Keywords: sexually transmitted infections; Helicobacter pylori; vagina; oral-genital contact

\section{Introduction}

Helicobacter pylori is the causal organism responsible for chronic gastritis and peptic ulcer disease. A recent meta-analysis of studies showed that the infection is also associated with a twofold increased risk of developing gastric cancer. ${ }^{1} H$ pylori infection has also been associated with a number of extragastric infections. ${ }^{2}$

The route of transmission of $H$ pylori infection has been widely hypothesised but the exact mode(s) of transmission is still unknown. Currently, several routes of transmission have been postulated including oral-oral, faecaloral, iatrogenic, fomite, and vector borne, but no definite transmission pathway(s) has yet been identified. In addition to the human stomach, the potential reservoirs of $H$ pylori include faeces, the cheek, saliva, and dental plaques. Animals, vomitus, water, and food are also candidates. ${ }^{3}$ These potential reservoirs are obviously important when trying to determine possible routes of transmission of this fastidious organism.

There have been many reviews that have been published looking at possible transmission routes and reservoirs of $H$ pylori. ${ }^{3-5}$ However, no published papers have examined the possibility of sexual transmission via the vagina. The aim of this paper is specifically to postulate the idea that $H$ pylori may be transmitted sexually and that the vagina may act as a reservoir.

\section{Hypothesis}

I would like to postulate that $H$ pylori infection could be transmitted sexually with the vagina acting as a potential temporary/permanent res-

Department of Medicine, University of Sydney, Nepean Hospital, Clinical Sciences Building, PO Box 63, Penrith, NSW 2751, Australia

eslickg@med.usyd.edu.au

Accepted for publication 29 June 2000 Epidemiological evidence

The natural acquisition of $H$ pylori infection appears to occur mostly during childhood and is associated with low socioeconomic status and poor hygiene. Faecal-oral transmission may play an important part in this acquisition. However, the mode of transmission among young adults and couples who continue to be infected, although at a low rate even with improved understanding and level of hygiene, remains to be elucidated. ${ }^{6}$ The possibility of sexual transmission via the vagina in adults would contribute, in part, to the low rates.

Previous data have suggested that sexual behaviour may be important in the transmission of $\mathrm{H}_{\text {pylori. }}{ }^{7-9}$ The majority of these studies have concentrated on the possibility of oroanal transmission between male homosexuals.

Seroprevalence studies have shown that in cohabiting couples with a partner who is infected with $H$ pylori the non-infected individual has an increased risk of contracting the infection. Studies have shown that prevalence rates were statistically significant between couples with and without $H$ pylori infection $(83.3 \%$ v $28.5 \%$ ) respectively. ${ }^{10}{ }^{11}$

Ethnicity may also be an important predictor of sexual transmission of $H$ pylori infection. A number of studies have shown that the highest rates of sexually transmitted infections occur in ethnic minorities (Australian Aborigines, African-Americans, Hispanics, etc ...). ${ }^{12}{ }^{13}$ The high prevalence of sexually transmitted infections correlates well with the high $H$ pylori prevalence rates that exist among these ethnic groups. ${ }^{513}$ It must also be remembered that there are differences in vaginal flora among different ethnic groups. This may in part be due to genetic or behavioural differences leading to altered vaginal flora. Sexual mixing (that is, mixing partners between ethnic groups) may increase the chance of transmission of $H$ pylori between partners from a high prevalence ethnic group (for example, African American) to a lower prevalence ethnic group (EuropeanAmerican).

Molecular studies have produced evidence

of $H$ pylori transmission between spouses. Schutze et $a l^{11}$ found that reinfection had been caused by the same $H$ pylori strain and identified the spouses of the patients as carriers of the identical strain. This was supported by other studies. ${ }^{14-16}$ Moreover, it has been shown 
that multiple strains of $H$ pylori may infect the same individual. ${ }^{17} 18$

Vertical transmission may also occur during birth if $H$ pylori is present in the vagina. Studies have shown that the prevalence of $H$ pylori in pregnant women is about $20 \% .{ }^{19}{ }^{20}$ In one case report, $H$ pylori was isolated 6 days after birth from a neonate who had developed frequent vomiting, weight loss, and difficulty in suckling. ${ }^{21}$ Moreover, the case findings of ulcerative oesophagitis and antral micronodular gastritis in such a short time after birth were surprising based on the assumption of contamination. Is it possible that this neonate might have been infected in utero?

The question remains, why hasn't a Helicobacter species been recovered from the female vagina when so many vaginal swabs have been done to culture organisms like Neisseria gonorrhoea? One of the reasons may be that Helicobacter species are difficult to grow in culture because of its fastidious nature and the correct culture techniques have not been employed to grow it. For optimal growth the organism requires a microaerophilic environment (5-7\% oxygen); selective media (although it can grow on most types of media); temperature between $33^{\circ} \mathrm{C}$ and $40^{\circ} \mathrm{C} ; 98$ $100 \%$ humidity, and $2-7$ days' incubation. ${ }^{22} \mathrm{~A}$ second reason may be that in a routine laboratory, microbiologists look for certain organisms based on the clinical information provided and not "one off" organisms which they would more than likely consider to be "contaminates" or "not clinically significant."

\section{Preliminary experimental studies}

A few studies have been conducted in an attempt to isolate $H$ pylori from the vagina. Early attempts produced negative results. ${ }^{23} 24$

Recently, de-Argila et $a l^{25}$ conducted a study which attempted to find $H$ pylori in vaginal secretions by taking vaginal brushings and using polymerase chain reaction (PCR), culture, and Gram stain. However, the diagnostic methods used failed to detect $H$ pylori. This may be because the testing time might be important and the sampling methods used may not have been adequate.

In order to determine if any studies had been conducted looking at $H$ pylori and sexual transmission I conducted an extensive literature search using the major electronic databases, including Medline, Embase, Biological Abstracts, and Currents Contents (January 1983 to August 2000). The keywords used for the search included Helicobacter pylori (Campylobacter pylori and Campylobacter pyloridis), sexual transmission, sexually transmitted disease, sex workers, prostitutes, vagina, micro-organisms, and epidemiology. Additional manual searches were made using the reference lists from the selected articles to retrieve other papers relevant to the topic.

I found that no studies have been conducted to determine if the prevalence of $H$ pylori infection is increased in female sex workers when compared with the general population. This would be an important population to assess for sexual transmission of $H$ pylori.
However, a case report which was published a year before Warren and Marshall's now famous original paper on "spiral bacteria in the stomach" may have found strains of Helicobacter in a woman's vagina associated with vaginitis. The article reports finding commashaped rods (1-4 $\mu \mathrm{m}$ in length), with a characteristic corkscrew motility having between four and eight flagellae. ${ }^{26}$ Some of the organisms were cultured under microaerophilic conditions and cultured after 72 hours' incubation at $37^{\circ} \mathrm{C}$. The biochemical profile of these unknown organisms matched very closely with that of $H$ pylori, although definitive tests such as urease activity were not undertaken. Could this unknown organism have been a Helicobacter species?

\section{$H$ pylori in the vaginal niche}

$H$ pylori normally lives in the stomach of humans and has the unique ability to survive in extreme acidic conditions. Can $H$ pylori's ability to exist in such a niche enable it to live in other acidic environments? The acidic nature of the vaginal tract may provide an adequate milieu for the survival of $H$ pylori. Assuming that the acidic environment is adequate for $H$ pylori, the length of colonisation may be lifelong; however, this may depend on other vaginal flora (and sexually transmitted diseases) and how they interact with $H$ pylori, as well as physical removal of the organism during menstruation.

To consider that $H$ pylori would not grow in the vagina because it is not a microaerophilic environment would be incorrect. The vagina supports the growth of a number of microaerophilic organisms as both normal flora and during pathological processes (lactobacilli, Trichomonas vaginalis, Actinomyces israelii). ${ }^{27-29}$

Other factors that may affect the sexual transmission of $H$ pylori might include a particular sexual practice or the coexistence of other venereal diseases (which cause disruption of the normal integrity of skin and mucosa and local aggregation, as well as systemic stimulation, of lymphocytes). Moreover, the possibility of other coexisting infectious and nutritional conditions (increase the level of infectivity and shorten the interval or generation time).

\section{Symbiosis between $H$ pylori and yeast} Siavoshi et $a l^{30}$ showed that $H$ pylori has a unique symbiotic relationship with yeast where the yeast may protect $H$ pylori against adverse environmental conditions. $H$ pylori penetrates the yeast and resides in a vacuole where it can survive heat $\left(100^{\circ} \mathrm{C}\right.$ for 15 minutes), dehydration $\left(37^{\circ} \mathrm{C}\right.$ for 3 months), and chlorination (2 $\mathrm{ppm}$ ). This symbiotic relationship may prolong the viability of $H$ pylori and play a crucial part in sexual transmission.

It should also be remembered that, owing to the ubiquitous distribution of yeast, it also represents a significant proportion of the normal flora of the human female vagina and the external genitalia. ${ }^{31}{ }^{32}$ Studies have shown that the prevalence of yeasts and/or Candida albicans in the normal vagina of healthy, non-pregnant women is between $5 \%$ and 
$30 \% .^{33}$ Yeasts have also been isolated as normal flora from the human mouth with $75 \%$ of yeasts found in the mouth being $C$ albicans, with other Candida sp making up the rest. ${ }^{34}$

The role of yeast in acting as a vector for the transmission of $H$ pylori may be a significant one owing to the fact that vulvovaginal candidiasis is a common condition and 10 $20 \%$ of women have an asymptomatic yeast infection. ${ }^{35}$

Moreover, bacterial vaginosis affects 25$35 \%$ of adult women and $\sim 50 \%$ of lesbians (who may be a high risk group with some studies stating that bacterial vaginosis may be easily sexually transferred between lesbians). ${ }^{36}{ }^{37}$ Further research needs to be undertaken to elucidate the mechanisms of transmission among lesbians and to determine if any species of Helicobacter play a part in bacterial vaginosis.

\section{Proposed transmission mode}

Transmission to the female genital tract would occur during sexual activities such as orogenital sex. Because $H$ pylori has been found and cultured from the saliva and mouth there exists a possibility that $H$ pylori may be transmitted from infected person to noninfected person via kissing or other sexual activities. ${ }^{38} 39$

Namavar et $a l^{\beta 9}$ detected $H$ pylori from the saliva, tongue, palate, and cheek. Normally, $H$ pylori colonises the columnar epithelium of the stomach, but if $H$ pylori can survive on the squamous epithelium in the mouth, then is it not possible that it may also inhabit the squamous epithelium of the vaginal tract? Anatomical changes such as cervical ectropion (columnar epithelium on the exocervix) may enhance the possibility of $H$ pylori colonisation. ${ }^{40}$

Fisk and Riley ${ }^{41}$ presented a case where a common oral bacterium (Group A $\beta$ haemolytic streptococci) was transmitted during fellatio causing pathology. Some individuals may permanently harbour $H$ pylori in their mouth, which may act as a reservoir for future reinfection. There are conflicting studies with regard to the importance of the oral cavity in the transmission of $H$ pylori infection. ${ }^{42}$

$H$ pylori may then colonise or pseudocolonise the vagina (it may not be necessary for $H$ pylori to attach itself to the vaginal epithelium); however, it may survive in the moist mucus which envelopes the vaginal space. Transmission may be made without penetrant sexual intercourse. Previous studies have all concentrated on the possibility that transmission occurs because of penetrant sex.

The time $H$ pylori remains viable in the vagina may vary, however, for those individuals who engage in sexual activities more frequently (such as female sex workers); the risk of transmission is greater even if $H$ pylori is not viable for long periods of time.

The natural infectious dose of $H$ pylori is unknown; however, based on the epidemiology of other enteric organisms, it would suggest that the infectious dose of $H$ pylori is small. ${ }^{43}$ Moreover, the infective dose provides a greater understanding of the mechanism of transmission and thus if the infective dose for $\mathrm{H}$ pylori is small, this would suggest spread via person to person.

Because of the anatomy of the female genitalia and the close proximity to the anus, one must also consider the possibility of a "red herring" where Helicobacter in the faeces could be transmitted by hand contact with the perineum or during "wiping" after defaecation.

POSSIBLE METHODS OF SEXUAL TRANSMISSION OF HELICOBACTER PYLORI

(1) Oro-anal (rimming)

(2) Oro-genital, direct transmission (cunnilingus)

(3) Oro-genital, indirect transmission (fellatio followed by sexual intercourse)

(4) Oro-oral, kissing (erotic)

(5) Masturbation (using infected saliva as a lubricant)

(6) Fomites (sex toys).

Once the organism has established a niche in the vagina it may be transmitted again from the vagina to the mouth of a non-infected male or female during oral sex or via intercourse which may then be followed by oral sex with that partner. The potential pathways are only as diverse as a person's sexual practices.

Because of an increasing world population, especially of younger age groups, increasing travel, and mixing of populations and the alteration of social values and increasing promiscuity there has been an astronomical increase in sexually transmitted diseases over the past four decades. ${ }^{44}$

\section{Future research}

If $H$ pylori could exist in such a niche as the female genital tract, would the organism produce any pathology? Would infection produce similar pathology as seen in an infected stomach - that is, ulcers, atrophy, cancer? How would we diagnose and treat such cases?

Further studies would be needed to determine the exact mechanisms by which $H$ pylori might be transmitted. If the female genital tract could be a possible and plausible reservoir for $H$ pylori, then these studies would concentrate on the individuals who are more sexually active and more likely to have multiple partners. Also, an appropriate sample size and adequate sampling methods should be applied.

Note added at proof stage

An updated search of the literature was made in August 2000.

I wish to thank Professor Nicholas J Talley, Dr Harry H-X Xia and Dr Harold M Lukse for their review of the manuscript and Lisa Jane Young for assisting with the literature search and collection of published articles. I also wish to thank Dr Allen Black $\mathrm{Jr}$ for providing scientific information and published articles on yeast infections and Geoff Griffin for protocols and culture techniques for isolating vaginal organisms. Finally, I would like to thank Dr Tong Liang for his writing this paper.

interest: None.

Funding: None.

1 Eslick GD, Lim LL-Y, Byles JE, et al. Association of Helicobacter pylori infection with gastric carcinoma: a metaanalysis. Am f Gastroenterol 1999;94:2373-9.

2 Tsang KW, Lam S-K. Helicobacter pylori and extradigestive diseases. F Gastroenterol Hepatol 1999;14:844-50.

Sahay PS, Axon ATR. Reservoirs of Helicobacter pylori and modes of transmission. Helicobacter 1996;1:175-82. 
4 Cave DR. How is Helicobacter pylori transmitted? Gastroenterology 1997;113:S9-14.

5 Goodman KJ, Correa P. The transmission of Helicobacter pylori. A critical review of the evidence. Int $\mathcal{F}$ Epidemiol

6 Xia HH-X, Talley NJ. Natural acquisition and spontaneous elimination of Helicobacter pylori infection: clinical implications. Am f Gastroenterol 1997;92:1780-7.

7 Aceti A, Attanasio R, Pennica A, et al. Campylobacter pylori infection in homosexuals. Lancet 1987; ii: 154-5.

8 Polish LB, Douglas JM, Davidson AJ, et al. Characterisation of risk factors for Helicobacter pylori infection among men attending a sexually transmitted disease clinic: lack of evidence for sexual transmission. F Clin Microbiol 1991;29: 2139-43.

9 Perez-Perez GI, Witkin SS, Dacker MD, et al. Seroprevalence of Helicobacter pylori infection in couples. $\mathcal{F}$ Clin Microbiol 1991;29:642-4.

10 Singh V, Trikha B, Vaiphel K, et al. Helicobacter pylori: evidence for spouse-to-spouse transmission. $\mathcal{f}$ Gastroenterol dence for spouse-to-spo

11 Schutze K, Hentschel E, Dragosics B, et al. Helicobacter pylori reinfection with identical organisms: transmission by the parents' spouses. Gut 1995;36:831-3.

12 Zenilman JM. Ethnicity and sexually transmitted infections. Curr Opinion Infect Dis 1998;11:47-52.

13 Mollison LC, Lecons RJ, Thein-Htut, et al. Upper gsatrointestinal endoscopy in central Australian aborigines. Med $\mathcal{F}$ Aust 1994;160:182-4.

14 Bamford KB, Bickley J, Collins JSA, et al. Helicobacter pylori: comparison of DNA fingerprints provides evidence for intrafamilial infection. Gut 1993;34:1348-50.

15 Parente F, Maconi G, Sangaletti O, et al. Prevalence of Helicobacter pylori and related gastroduodenal lesions in spouses of Helicobacter pylori positive patients with duodenal ulcer. Gut 1996;39:629-33.

16 Georgopoulos SD, Mentis AF, Spiliadis CA, et al. Helicobacter pylori infection in spouses of patients with duodenal ulcers and comparison of ribosomal RNA gene duodenal ulcers and comparison

17 Jorgensen $\mathrm{M}$, Daskalopoulos G, Warburton V, et al. Multiple strain colonization and metronidazole resistance in Helicobacter pylori-infected patients-indentification from sequential and

18 Hirschl AM, Richter M, Makristathis A, et al. Single and multiple strain colonoization in patients with Helicobacter pylori-associated gastritis: detection by macrorestriction DNA analysis. F Infect Dis 1994;170:473-5.

19 Blecker U, Lanciers S, Keppens E, et al. Evolution of Helicobacter pylori positivity in infants born from positive mothers. F Pediatr Gastroenterol Nutr 1994;19:87-90.

20 Yan P, Eslick GD, Xia HH-X, et al. Association between Helicobacter pylori infection and fetal intrauterine growth retardation (IUGR). Gastroenterology 2000;118(suppl 2): A734.

21 Raymond J, Baragoui K, Kalach N, et al. Isolation of Helicobacter pylori in a six-day old newborn. Eur 7 Clin Microbiol Infect Dis 1995;14:727-8.

22 Goodwin CS, Blincow E, Warren JR, et al. Evaluation of cultural techniques for isolating Campylobacter pyloris from endoscopic biopsies of gastric mucosa. 7 Clin Path 1985;138:1127-31.

23 Marshall BJ, McGechie DB, Rogers PA, et al. Pyloric Campylobacter infection and gastroduodenal disease. Med $\mathcal{F}$ A ust 1985;142:439-44.

24 Eldridge J, Jones DM, Sethi P. The occurrence of antibody to the Campylobacter pylordis in various groups of individuals. In: Pearson AD, Skirrow MB, Lior H, Rowe B, eds. Camplyobacter III. Proceedings of the Third Interational Workshop on Campylobacter infections. London: PHLS, 1985:183-4.

25 Martin-de-Argila C, Arata IG, Boixeda D, et al. Helicobacter pylori at vaginal secretions. Gastroenterology 1998; 14:A217.

26 Holst E, Skarin A, Mardh PA. Characteristics of anaerobic comma-shaped bacteria recovered from the female genital tract. Eur f Clin Microbiol 1982;1:310-16.

27 Reid G, McGroarty RG, Tomeczek L, et al. Identification and plasmid profiles of Lactobacillus species from the vagina of 100 healthy women. FEMS Immunol Med Microbiol 1996;15:23-6.

28 Ellis JE, Yarlett N, Cole D, et al. Antioxidant defences in the microaerophilic protozoan Trichomonas vaginalis: comparison of metronidazole-resistant and sensitive strains. Microbiology 1994:140:2489-94.

29 Pine L, Malcom GB, Curtis EM, et al. Demonstration of Actinomyces and Arachnia species in cervicovaginal smears by direct staining with species-specific fluorescentantibody conjugate. $\mathcal{F}$ Clin Microbiol 1981;13:15-21.

30 Siavoshi F, Nourali-Ahari F, Zeinali S, et al. Yeast a silent companion of $\mathrm{H}$ pylori which protects it against the environmental stresses. Gastroenterology 1996;110:A1015.

31 Rippon JW.. Medical mycology: the pathogenic fungi and the pathogenic actinomycetes. 2nd ed. London: WB Saunders, 1982:490

32 Murray PR, et al, ed. Manual of clinical microbiology. 7th ed. Washington DC: American Society for Microbiology, 1999:23-32

33 Linares de LM, Martin C. Frequency of yeasts of the genus Candida in humans as pathogens and as part of normal flora. In: The black yeasts and the white yeasts. Proceedings of the IV International Conference on the Mycoses. Pan American Health Association Washington Sci Pub No 356, 1978:124-36.

34 Martin MV, Wilkinson GR. The oral yeast flora of 10-yearold schoolchildren. Sabouraudia 1983;21:129-35.

35 Zdolsek B, Hellberg D, Froman G, et al. Vaginal microbiological flora and sexually transmitted diseases in women gith recurrent or current vulvovaginal candidiasis. Infection 1995;2:81-4.

36 Berger BJ, Kolton S, Zenilman JM, et al. Bacterial vaginosis in lesbians: a sexually transmitted disease. Clin Infect Dis 1995;21:1402-5.

37 Edwards A, Thin RN. Sexually transmitted diseases in lesbians. Int f STD AIDS 1990;1:178-81.

38 Ferguson DA, Li C, Patel NR, et al. Isolation of Helicobacter pylori from saliva. F Clin Microbiol 1993;31: 2802-4.

39 Namavar F, Roosendaal R, Kuipers EJ, et al. Presence of Helicobacter pylori in the oral cavity, oesophagus, stomach, and faeces of patients with gastritis. Eur 7 Clin Microbiol Infect Dis 1995;14:234-7.

40 Coleman DV, Evans DMD. Biopsy pathology and cytology of the cervix. London: Chapman and Hall, 1988:35-40; 63-5.

41 Fisk P, Riley V. Fellatio in the sexual transmission of Lancefield Group A $\beta$-haemolytic streptococcus. Int F STD AIDS 1995;6:458.

42 Thomas E, Jiang C, Chi DS, et al. The role of the oral cavity in Helicobacter pylori infection. Am $\mathcal{f}$ Gastroenterol 1997;92:2148-54.

43 Feldman RA, Eccersley AJP, Hardie JM. Epidemiology of Helicobacter pylori: acquisition, transmission, population prevalence and disease-to-infection ratio. $\mathrm{Br}$ Med Bull 1998;54:39-53

44 Webber R. Communicable disease epidemiology and control. London: CAB International, 1997:217. 